\title{
Hadronic structure from the lattice
}

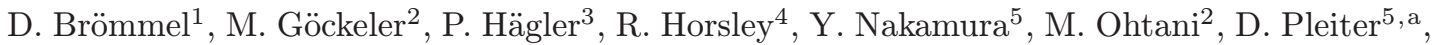
P.E.L. Rakow ${ }^{6}$, A. Schäfer ${ }^{2}$, G. Schierholz ${ }^{5}$, W. Schroers ${ }^{7}$, H. Stüben ${ }^{8}$, and J.M. Zanotti ${ }^{4}$

1 School of Physics and Astronomy, University of Southampton, Southampton SO17 1BJ, UK

2 Institut für Theoretische Physik, Universität Regensburg, 93040 Regensburg, Germany

3 Institut für Theoretische Physik T39, TU München, 85747 Garching, Germany

4 School of Physics, University of Edinburgh, Edinburgh EH9 3JZ, UK

5 Deutsches Elektronen-Synchrotron DESY and John von Neumann Institut für Computing NIC, 15738 Zeuthen, Germany

6 Theoretical Physics Division, Department of Mathematical Sciences, University of Liverpool, Liverpool L69 3BX, UK

7 Department of Physics, Center for Theoretical Sciences, National Taiwan University, Taipei 10617, Taiwan

8 Konrad-Zuse-Zentrum für Informationstechnik Berlin, 14195 Berlin, Germany

Abstract. In recent years the investigation of hadron structure using lattice techniques has attracted growing attention. The computation of several important quantities has become feasible. Furthermore, theoretical developments as well as progress in algorithms and an increase in computing resources have contributed to a significantly improved control of systematic errors. In this article we give an overview on the work that has been carried out in the framework of the Hadron Physics I3 (I3HP) network "Computational (lattice) hadron physics". Here we will not restrict ourselves to spin physics but focus on results for nucleon spectrum and structure from the QCDSF collaboration. For a broader overview of developments in this field see, e.g., [1].

\section{Introduction}

The main goal of lattice hadron phenomenology is the computation of generalized parton distributions (GPDs) of mesons and nucleons from first principles. GPDs have become a theoretical framework which enables us to study many fundamental aspects of the intrinsic hadron structure. It allows us to confront experimental results and lattice calculations since it includes as limiting cases hadron form factors as well as polarized and unpolarized parton densities, i.e. quantities which are investigated by various experiments at CERN (COMPASS), DESY (H1, Zeus, Hermes) and JLab. Combining information from both experiment and lattice offers new opportunities to explore hadrons as extended objects.

On the lattice we are restricted to the calculation of moments of GPDs. As an example we consider the unpolarized GPDs $H^{q}\left(x, \xi, Q^{2}\right)$ and $E^{q}\left(x, \xi, Q^{2}\right)$. Their moments are related to the generalized form factors (GFFs) $A_{n, k}^{q}\left(Q^{2}\right), B_{n, k}^{q}\left(Q^{2}\right)$ and $C_{n}^{q}\left(Q^{2}\right)$ :

$$
\int_{-1}^{+1} d x x^{n-1}\left[\begin{array}{c}
H^{q}\left(x, \xi, Q^{2}\right) \\
E^{q}\left(x, \xi, Q^{2}\right)
\end{array}\right]=\sum_{i=0}^{\left[\frac{n-1}{2}\right]}\left[\begin{array}{c}
A_{n, 2 i}^{q}\left(Q^{2}\right) \\
B_{n, 2 i}^{q}\left(Q^{2}\right)
\end{array}\right](-2 \xi)^{2 i} \pm \operatorname{Mod}(n+1,2) C_{n}^{q}\left(Q^{2}\right)(-2 \xi)^{n} .
$$

\footnotetext{
${ }^{\mathrm{a}}$ e-mail: dirk.pleiter@desy.de
} 
The GFFs can be calculated from a parametrization of (nucleon) matrix elements. As an illustrative example one may consider the GFFs $A_{1,0}^{q}$ and $B_{1,0}^{q}$ :

$$
\left\langle p^{\prime}, s^{\prime}\left|\bar{u} \gamma^{\mu} u\right| p, s\right\rangle=\bar{u}\left(p^{\prime}, s^{\prime}\right)\left\{\gamma^{\mu} A_{1,0}^{(u)}\left(Q^{2}\right)+i \sigma^{\mu \nu} \frac{q_{\nu}}{2 m_{N}} B_{1,0}^{(u)}\left(Q^{2}\right)\right\} u(p, s),
$$

where $p(s)$ and $p^{\prime}\left(s^{\prime}\right)$ denote initial and final momenta (spins), $q=p^{\prime}-p$ the momentum transfer (with $Q^{2}=-q^{2}$ ). Instead of calculating the matrix elements (i.e., the 1.h.s. of Eq. (2)) in Minkowski space-time, we calculate these within a discretized Euclidean space-time by using appropriate combinations of two- and three-point correlation functions. A typical combination is the following ratio:

$$
R\left(t, \tau, p^{\prime}, p\right)=\frac{C_{3}\left(t, \tau, p^{\prime}, p\right)}{C_{2}\left(t, p^{\prime}\right)} \times\left[\frac{C_{2}\left(\tau, p^{\prime}\right) C_{2}\left(t, p^{\prime}\right) C_{2}(t-\tau, p)}{C_{2}(\tau, p) C_{2}(t, p) C_{2}\left(t-\tau, p^{\prime}\right)}\right]^{1 / 2}
$$

where $C_{2}(t, p)$ is the unpolarized two-point function with a source at time 0 and sink at time $t$, while the three-point function $C_{3}\left(t, \tau, p^{\prime}, p\right)$ has an (local) operator $\mathcal{O}$ insertion at time $\tau$. The ratio $R\left(t, \tau, p^{\prime}, p\right)$ is expected to become constant when keeping sufficiently far away from source and sink, i.e. for $0 \ll \tau \ll t \lesssim \frac{1}{2} L_{\mathrm{T}}$. For further details see, e.g., [2].

\subsection{Lattice obstacles}

All lattice calculations are faced with the problem of keeping their systematic errors under control. The main sources for such systematic errors are the following:

- In almost all cases lattice results have to be extrapolated to the

- infinite volume limit: $V \rightarrow \infty$.

- continuum limit: $a \rightarrow 0$ where $a$ is the lattice spacing.

- region of light quark masses: $m_{\mathrm{ps}} \rightarrow m_{\pi}$.

- Renormalization and mixing of operators.

- Conversion of the lattice results into physical units.

The results which we present in this article have been obtained from simulations with $N_{\mathrm{f}}=2$ flavours of dynamical $O(a)$-improved Wilson fermions (so-called Clover fermions). Improvement of both the action and the operators is expected to bring discretization errors down to $O\left(a^{2}\right)$. Our simulations have been performed with lattice spacings in the range of 0.11 down to $0.07 \mathrm{fm}$. For most of our results we find discretization effects to be small and often negligible compared to, e.g., statistical errors.

For some of the observables considered here, finite volume effects turned out to be large. In our simulations the lattices have a spatial extension in the range $L_{s}=1.4, \ldots, 2.6 \mathrm{fm}$. Results from chiral effective field theories may be used to estimate or even correct for finite size effects.

Typically, the largest uncertainty originates from the extrapolation of the results down to the region of physical light quark masses. Results presented here have been obtained for pseudoscalar meson masses in the range of $300 \mathrm{MeV}$ up to $1 \mathrm{GeV}$. Due to a combination of significantly improved algorithms (see [3] for a recent review on algorithms for simulations with dynamical fermions) and the availability of increased computer power it has become possible to start exploring a quark mass region where one may hope results from chiral perturbation theory to be applicable.

Another source of systematic errors stems from renormalization. Perturbative renormalization techniques suffer from their complexity when going beyond 1-loop and, more importantly, from their poor convergence. It turned out to be crucial to employ non-perturbative renormalization techniques. As an illustrative example we compare in Fig. 1 (left panel) the vector-current renormalization constant $Z_{V}$ obtained from 1-loop perturbation theory and a non-perturbative determination, where we imposed as renormalization condition the equivalence of the local and the conserved vector current (see [4] for a detailed description of this method). 

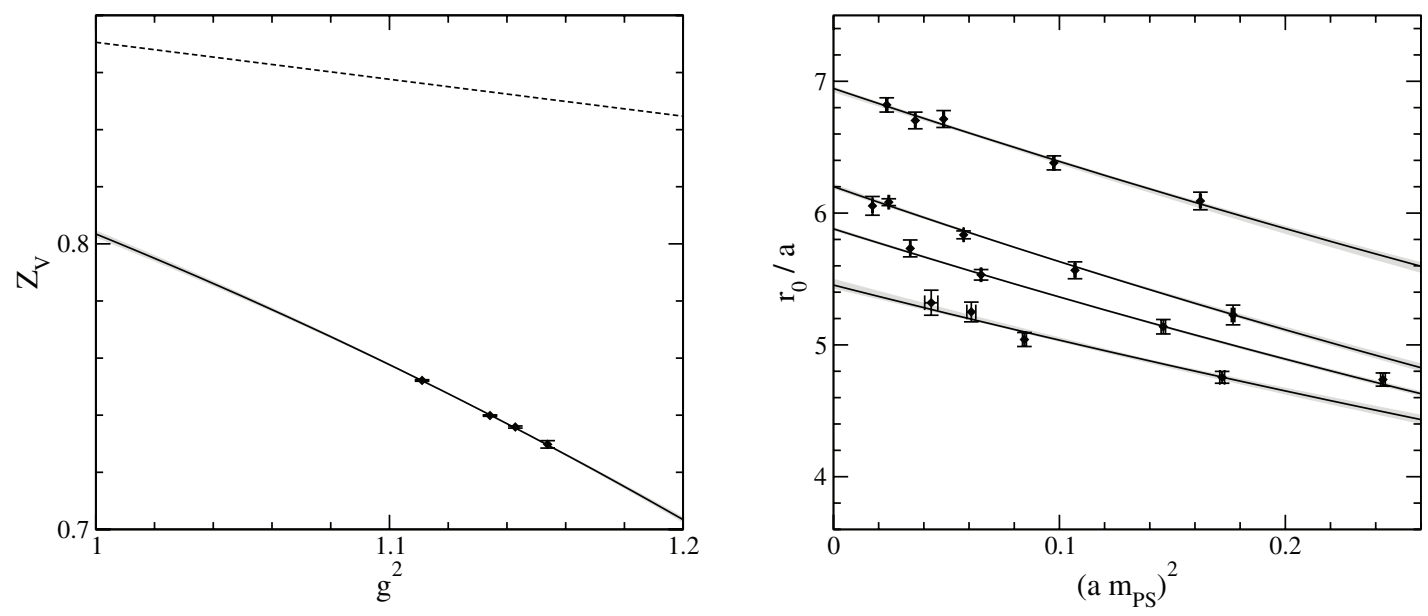

Fig. 1. The left plot shows a comparison of $Z_{V}$ obtained from 1-loop perturbation theory (dashed line) and a non-perturbative determination (solid line). The right plot shows the values for $r_{0}\left(\beta, m_{\mathrm{ps}}\right)$ $(\beta=5.20,5.25,5.29,5.40)$ together with an extrapolation to the chiral limit.

Let us finally consider the problem of converting lattice results into physical units. It has become popular to use the Sommer parameter $r_{0}$, which is defined by the expression $r_{0}^{2} F\left(r_{0}\right)=$ 1.65. The force $F(r)$ can be determined with rather high statistical accuracy from the static quark potential. This strategy however suffers from the problem that the phenomenological value is not very well known. Typically, $r_{0} \simeq 0.5 \mathrm{fm}$ is used. Lattice results, e.g., for the nucleon mass (see [5]), suggest a significantly smaller value. Here we will use $r_{0}=0.467 \mathrm{fm}[6,7]$. Fig. 1 (right panel) shows our results for $r_{0}$.

\section{Masses}

As a first example for using chiral effective field theories in order to guide extrapolation to physical quark masses we consider the mass of the nucleon, $m_{\mathrm{N}}$. Calculations using relativistic baryon chiral perturbation theory $(\mathrm{B} \chi \mathrm{PT})$ suggest a rather non-trivial quark mass dependence. The $p$-expansion at $O\left(p^{4}\right)$ in an infinite volume gives the following quark mass dependence [8]:

$$
\begin{aligned}
& m_{\mathrm{N}}\left(m_{\mathrm{ps}}\right)=M_{0}-4 c_{1} m_{\mathrm{ps}}^{2}-\frac{3 g_{A, 0}{ }^{2}}{32 \pi F_{0}^{2}} m_{\mathrm{ps}}^{3} \\
& +\left[e_{1}^{r}(\lambda)-\frac{3}{64 \pi^{2} F_{0}^{2}}\left(\frac{g_{A, 0}{ }^{2}}{M_{0}}-\frac{c_{2}}{2}\right)-\frac{3}{32 \pi^{2} F_{0}^{2}}\left(\frac{g_{A, 0}{ }^{2}}{M_{0}}-8 c_{1}+c_{2}+4 c_{3}\right) \ln \frac{m_{\mathrm{ps}}}{\lambda}\right] m_{\mathrm{ps}}^{4} \\
& +\frac{3 g_{A, 0}{ }^{2}}{256 \pi{F_{0}}^{2} M_{0}^{2}} m_{\mathrm{ps}}^{5}+O\left(m_{\mathrm{ps}}^{6}\right) .
\end{aligned}
$$

Even using all our lattice measurements at quark masses in the the range $0<m_{\mathrm{ps}} \lesssim 650 \mathrm{MeV}$ it is not possible to sufficiently constrain all parameters in Eq. (4). We therefore reduce the number of free fit parameters to the nucleon mass in the chiral limit $M_{0}$, the not very well known low-energy constant (LEC) $c_{1}$ and the counter-term $e_{1}^{r}(\lambda)$ (we use $\lambda=1 \mathrm{GeV}$ ). For all other parameters, i.e. the LECs $c_{2}$ and $c_{3}$, the pion decay constant $F_{0}$ and the nucleon axial coupling $g_{A, 0}$, we use phenomenological estimates. Both lattice data and the resulting fit are shown in Fig. 2 (left panel). The lattice data seems to fall on a universal curve indicating discretisation effects to be small, which we thus ignored. We observe that $m_{\mathrm{N}}\left(m_{\mathrm{ps}}=m_{\pi}\right)$ is consistent with experiment. Furthermore, we find $c_{1}=-1.02(7) \mathrm{GeV}^{-1}$, a value which is consistent with other estimates [9]. 

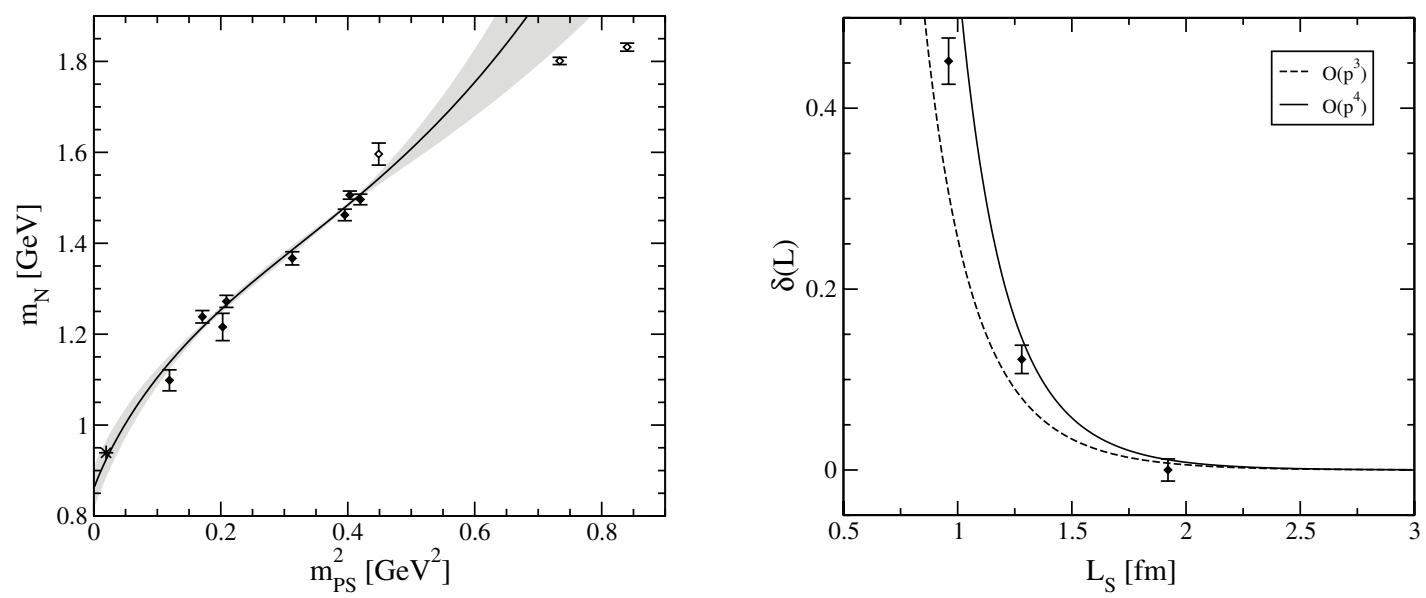

Fig. 2. The left plot shows lattice results for $m_{\mathrm{N}}$ together with a fit to Eq. (4). The star indicates the physical point. In the right plot we compare the relative finite size effect $\delta(\mathrm{L})=\left(m_{N}(\mathrm{~L})-\right.$ $\left.m_{N}(\infty)\right) / m_{N}(\infty)$ as measured on the lattice and predicted by $\mathrm{B} \chi \mathrm{PT}$. The diamonds show results from lattices with $V=12^{3} \times 32,16^{3} \times 32,24^{3} \times 48$ (left to right).

$\mathrm{B} \chi \mathrm{PT}$ has also been used to calculate the finite size effects [6]:

$$
m_{N}\left(L_{s}\right)-m_{N}(\infty)=\Delta_{a}\left(L_{s}\right)+\Delta_{b}\left(L_{s}\right)+O\left(p^{5}\right),
$$

where $\Delta_{a}$ represents the $O\left(p^{3}\right)$ result for the volume dependence and $\Delta_{b}$ additional contributions at $O\left(p^{4}\right)$. All coefficients in $\Delta_{a}\left(L_{s}\right)$ and $\Delta_{b}\left(L_{s}\right)$ are also present in Eq. (4). It is thus possible to estimate the finite volume effects obtained from Eq. (5) using the coefficients obtained from a fit to Eq. (4). These estimates can then be used for a comparison with values of the nucleon mass computed on lattices that have a smaller (physical) volume. Fig. 2 (right panel) shows such a comparison at fixed $m_{\mathrm{ps}} \simeq 590 \mathrm{MeV}$.

\section{Nucleon axial coupling}

Next, we consider the axial coupling constant $g_{A}=G_{A}(0)$, where $G_{A}\left(Q^{2}\right)$ is the axial form factor of the nucleon. It is determined from the renormalized axial vector current $A_{\mu}^{R}=Z_{A}(1+$ $\left.b_{A} a m_{q}\right) A_{\mu}$, where $a m_{q}$ is the bare quark mass. Here we only look into the iso-vector case where contributions of so-called disconnected terms cancel. These are contributions of diagrams which only interact with the hadron via the exchange of gluons and which are hard to compute on the lattice. While $Z_{A}$ is known non-perturbatively [10], $b_{A}$ is only known perturbatively and is computed using tadpole improved one-loop perturbation theory.

Like for the nucleon mass the quark mass dependence of the iso-vector nucleon axial coupling has been calculated using chiral effective field theory [10,11]. The calculations using the small scale expansion (SSE) have been performed in the infinite volume limit as well as for a finite spatial cubic box of length $L_{s}$. We define $g_{A}\left(m_{\mathrm{ps}}, L_{s}\right)=g_{A}\left(m_{\mathrm{ps}}\right)+\Delta g_{A}\left(m_{\mathrm{ps}}, L_{s}\right)$, where $\Delta g_{A}\left(m_{\mathrm{ps}}, L_{s}\right)$ denotes the finite size effects. Given the large number of parameters in the resulting expressions we again have to fix some of them using phenomenological input like the pion decay constant, the $N \Delta$ mass splitting and the axial $N \Delta$ coupling.

We find the finite size effects to be significantly larger compared to the nucleon mass. These effects can not be ignored and we have to resort to a fit which includes both quark mass dependence and finite size effects. In Fig. 3 we show results for the quark mass dependence in the infinite volume limit and finite size effects.

For results for $G_{A}\left(Q^{2}\right)$ at non-zero $Q^{2}$ and for the pseudo-scalar form factor $G_{P}\left(Q^{2}\right)$ see [12]. For an independent calculation of $g_{A}$ see [13]. 

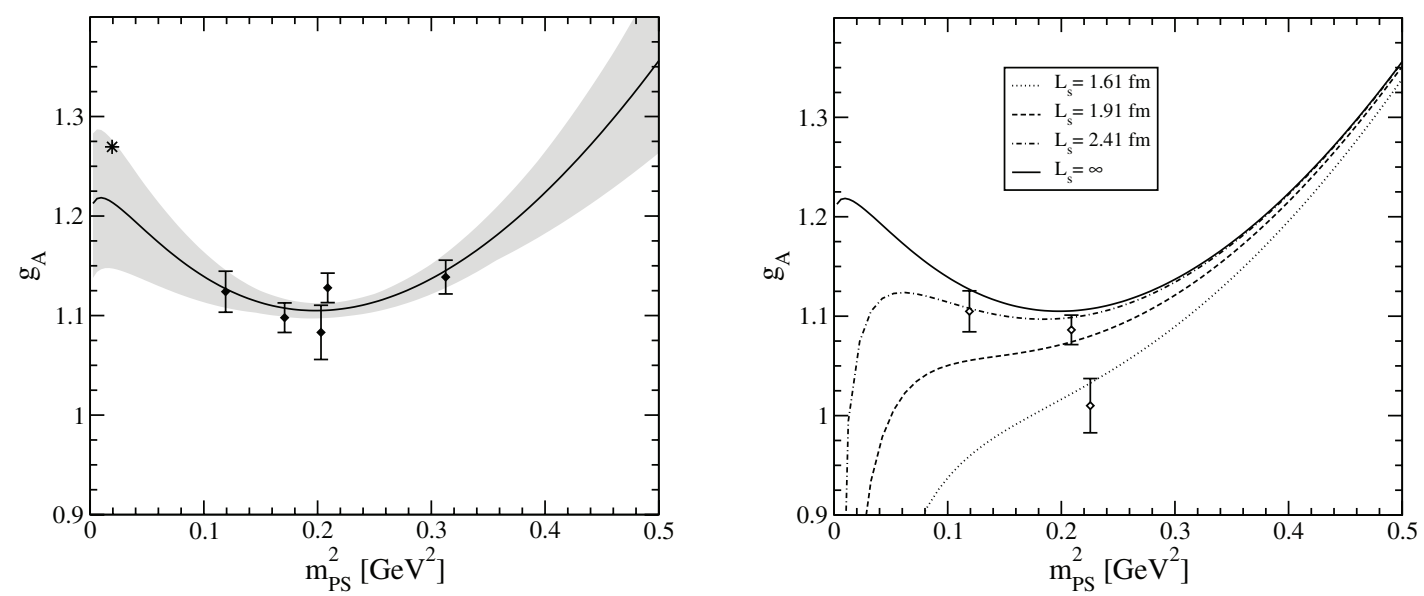

Fig. 3. The left plot shows $g_{A}\left(m_{\mathrm{ps}}\right)$ in the infinite volume limit and the right plot the data points before correcting the finite size effects.

\section{Electro-magnetic form factors}

JLab polarisation experiments $[14,15]$ have in recent years led to a revived interest in the nucleon electro-magnetic form factors. Measurements of the ratio of the proton electric to magnetic form factors, $\mu^{(\mathrm{p})} G_{e}^{(\mathrm{p})}\left(Q^{2}\right) / G_{m}^{(\mathrm{p})}\left(Q^{2}\right)$, showed an unexpected decrease. This means that the proton's electric form factor falls off faster than the magnetic form factor.

The form factors are obtained from the standard decomposition of the nucleon electromagnetic matrix elements

$$
\left\langle p^{\prime}, s^{\prime}\left|J^{\mu}\right| p, s\right\rangle=\bar{u}\left(p^{\prime}, s^{\prime}\right)\left[\gamma_{\mu} F_{1}^{(u)}\left(Q^{2}\right)+i \sigma^{\mu \nu} \frac{q_{\nu}}{2 m_{N}} F_{2}^{(u)}\left(Q^{2}\right)\right] u(p, s) .
$$

By calculating the matrix elements on the l.h.s. and the nucleon mass we obtain the Dirac form factor $F_{1}\left(Q^{2}\right)$ and the Pauli form factor $F_{2}\left(Q^{2}\right)$.

Particularly interesting are the $Q^{2}$ scaling and flavour dependence of the form factors. We find that the lattice data can be well parametrized using a pole ansatz

$$
F_{i}\left(Q^{2}\right)=\frac{A_{i}}{\left(1+Q^{2} / M_{i}^{2}\right)^{p}} .
$$

(For a different ansatz see [12].) Naively, one would expect $p=2$ for $F_{1}$ and $p=3$ for $F_{2}$. Our lattice data, however, has a flavour dependence which favours $p=2$ for $F_{1}^{(u)}$ and $p=3$ otherwise. In Fig. 4 (left panel) we plot the ratio $F_{1}^{(d)} / F_{1}^{(u)}$ which one naively would expect to be constant. This result should be taken with some care since disconnected contributions have not been calculated. These contributions only cancel in the iso-vector case. Our observation is however consistent with the flavour dependence observed in fits to experimental data [16].

For a more quantitative comparison with experimental data we have to extrapolate our results to physical quark masses. We again do this on the basis of results from chiral effective field theories using the SSE $[17,18]$. These calculations predict a strong quark mass dependence for the iso-vector form factor radii and the iso-vector anomalous magnetic moment in the small quark mass region. In Fig. 4 (right panel) we compare our results for the iso-vector Dirac radius $r_{2}^{(u-d)}$ and a fit to the SSE expression. The lattice results are significantly smaller than 

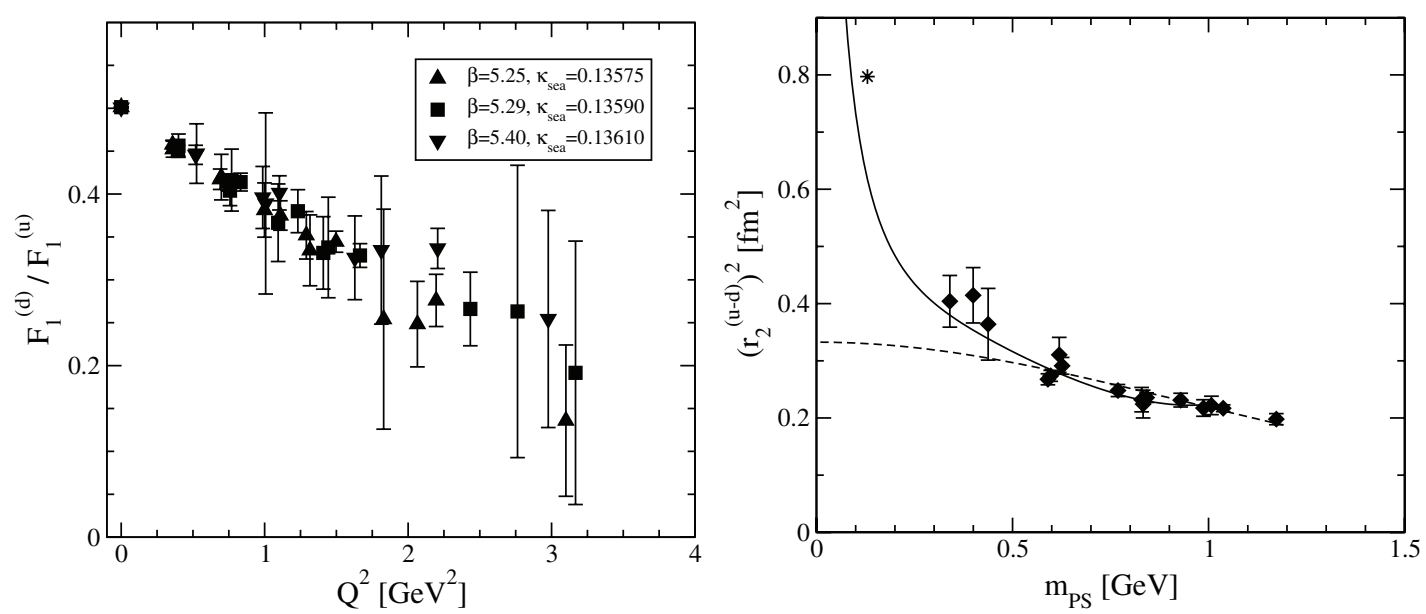

Fig. 4. The left plot shows the ratio $F_{1}^{(d)} / F_{1}^{(u)}$ as a function of $Q^{2}$ for different $\beta$ values but similar $m_{\mathrm{ps}} \simeq 400 \mathrm{MeV}$. The right plot shows the extrapolation of our results for the Dirac form factor radius $r_{2}^{(u-d)}$. The solid line shows a fit with the SSE expression, the dashed line a fit to an ansatz linear in the quark mass.

the experimental value. However, the SSE results indicate a strong quark mass dependence for $m_{\mathrm{ps}} \lesssim 250 \mathrm{MeV}$, a region which is currently difficult to access in lattice simulations.

\section{Moments of unpolarized structure functions}

As another quantity which can be calculated on the lattice we consider the lowest moment of the unpolarized nucleon structure function, $\langle x\rangle=A_{2,0}^{q}(0)$, where $A_{2,0}^{q}$ is the first moment of $H^{q}\left(x, \xi, Q^{2}\right)$ at $\xi=0$.

This moment is determined from the matrix element

$$
\left\langle p, s\left|\left[\mathcal{O}^{\left\{\mu_{1} \mu_{2}\right\}}-\operatorname{Tr}\right]\right| p, s\right\rangle=2 A_{2,0}^{q}\left[p^{\mu_{1}} p^{\mu_{2}}-\operatorname{Tr}\right],
$$

where $\mathcal{O}^{\left\{\mu_{1} \mu_{2}\right\}}=\bar{u} \gamma^{\mu_{1}} i \stackrel{\leftrightarrow}{D^{\mu_{2}}} u$. The renormalisation of this matrix element has been performed non-perturbatively by means of the RI'-MOM method [19]. In the perturbative conversion to $\overline{\mathrm{MS}}$ at a scale of $2 \mathrm{GeV}$ we have used $\Lambda^{\overline{\mathrm{MS}}}=261(17)(26) \mathrm{MeV}[20]$.

In the range of quark masses which is currently accessible the results in the iso-vector channel $\langle x\rangle^{(u-d)}$ are significantly larger than the experimental value. However, it has been suggested that this quantity may become significantly smaller at very light quark masses [21]. This has been confirmed by recent calculations in the framework of baryon chiral perturbation theory $(\mathrm{B} \chi \mathrm{PT})$ [22]. These recent calculations have been used to fit our results using the pion decay constant, the nucleon mass and the nucleon axial coupling in the chiral limit as phenomenological input. This leaves two free fit parameters, which allows us to fit the data for different values of $\beta$ separately restricting the fit range to $0<m_{\mathrm{ps}} \lesssim 650 \mathrm{MeV}$. In Fig. 5 we plot the fit to the $\beta=5.29$ data showing little evidence for $\langle x\rangle^{(u-d)}\left(m_{\mathrm{ps}}\right)$ becoming smaller for $m_{\mathrm{ps}} \rightarrow m_{\pi}$. In our results we find no indication for large discretisation effects (see right panel of Fig. 5). However, for the results at light quark masses finite size effects cannot be excluded.

In Fig. 6 (left) the $Q^{2}$ dependence of the generalized form factor $A_{2,0}^{q}$ is shown together with the other unpolarized GFFs $B_{2,0}^{q}$ and $C_{2}^{q}$. The lattice results for $A_{2,0}^{q}$ can be well described by a dipol expression $A_{2,0}^{q}\left(Q^{2}\right)=A_{2,0}^{q}(0) /\left(1+Q^{2} / M_{D}^{2}\right)^{2}$. The quark mass dependence of the dipole mass is also shown in Fig. 6 (right plot). For further details see [23]. 

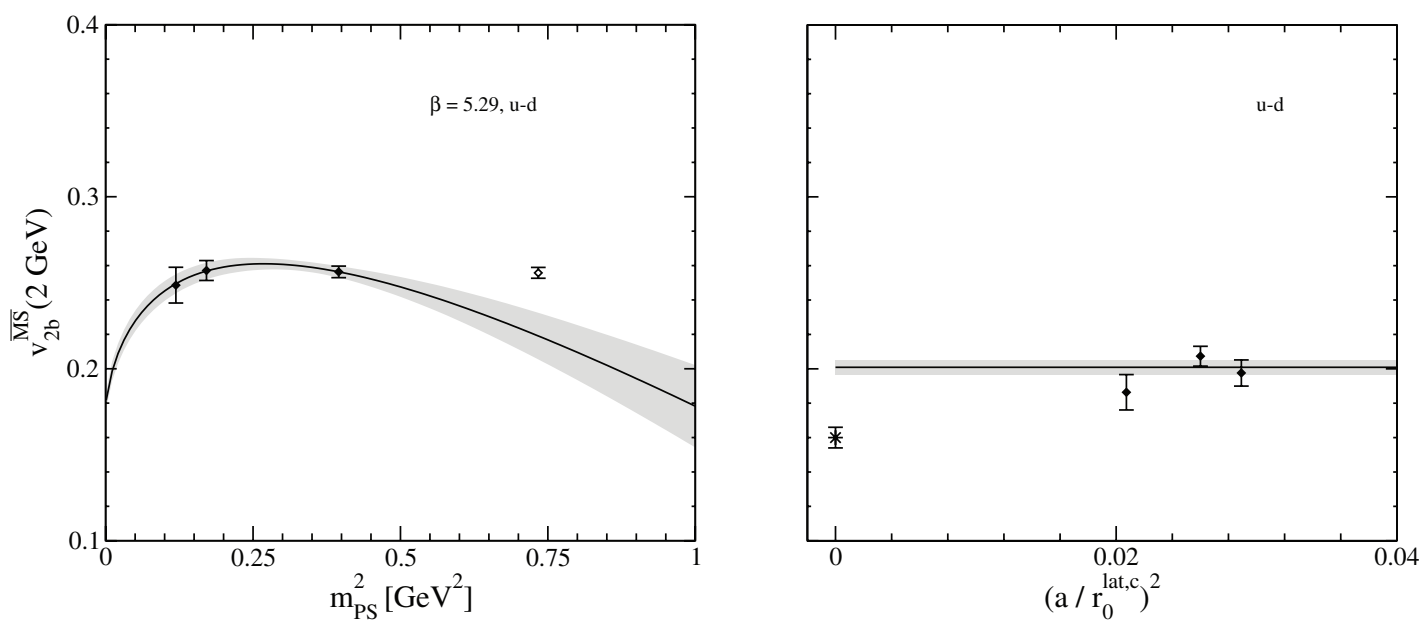

Fig. 5. The left plot shows our results for $\langle x\rangle^{(u-d)}\left(m_{\mathrm{ps}}\right)$ at $\beta=5.29$ (the results for other values of $\beta$ are similar). The solid line denotes a fit to a B $\chi \mathrm{PT}$ expression [22]. The right plot shows $\langle x\rangle^{(u-d)}\left(m_{\mathrm{ps}}=m_{\pi}\right)$ as a function of the lattice spacing together with a fit to a constant. The star denotes the phenomenological value from CTEQ6.
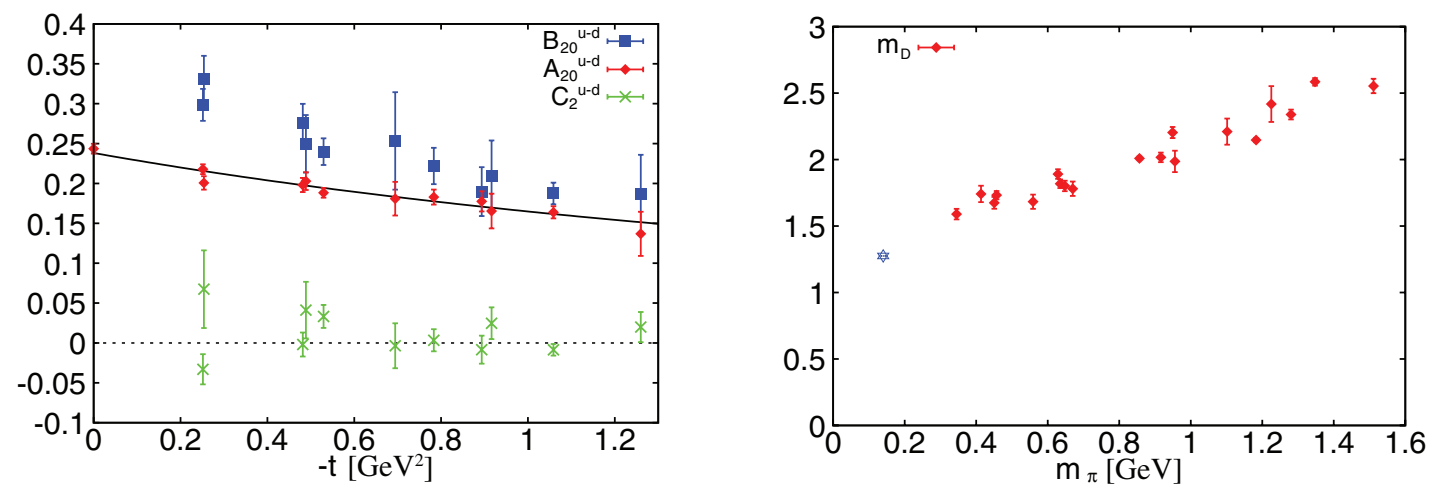

Fig. 6. The left plot shows the generalised form factors $A_{2,0}^{q}, B_{2,0}^{q}$ and $C_{2}^{q}$ in the iso-vector channel at $\kappa=0.13632$ and $\beta=5.29$ with $m_{\mathrm{ps}} \simeq 350 \mathrm{MeV}$. In the right plot the dipole masses of $A_{2,0}^{q}$ are shown as a function of the pseudo-scalar meson mass. The star represents the experimental value of the tensor meson mass $f_{2}$.

\section{Other results}

Several other results have been published in recent years, which we will summarize here only very briefly:

- A calculation of the lowest two moments of transverse spin densities of quarks in the nucleon has revealed strongly distorted densities of transversely polarized quarks in the nucleon [24].

- The electro-magnetic form factor $F_{\pi}$ of the pion calculated on the lattice has been found to be in good agreement with experimental results [25]. The charge radius squared was obtained as $\left\langle r^{2}\right\rangle=0.441(19) \mathrm{fm}^{2}$.

- Lattice calculations of the first two moments of the quark tensor GPD $E_{\mathrm{T}}^{\pi}$ indicate - like in the case of the nucleon - a strongly distorted spatial distribution of the quarks if they are transversely polarized [26]. 


\section{Conclusions and outlook}

In this talk we have presented a small selection of results to demonstrate where lattice calculations can contribute to the investigation of hadron structure. While these results may improve our qualitative understanding, for quantitatively precise results further efforts are needed and keeping systematic errors under control remains a major challenge. In particular, the necessary extrapolations to the infinite volume, vanishing lattice spacing and to the physical quark masses remain a major source of uncertainties. While results from chiral effective field theories help to guide these extrapolations, eventually simulations using large lattices and very light quark masses are needed. We conclude with the good news that such simulations are now becoming feasible.

The numerical calculations have been performed on the Hitachi SR8000 at LRZ (Munich), the Cray T3E at EPCC (Edinburgh) the APE1000 and apeNEXT at NIC/DESY (Zeuthen), the BlueGene/L at NIC/FZJ (Jülich) and EPCC (Edinburgh). Some of the configurations at the small pion mass have been generated on the BlueGene/L at KEK by the Kanazawa group as part of the DIK research programme. This work was supported in part by the DFG, by the EU Integrated Infrastructure Initiative Hadron Physics (I3HP) under contract number RII3-CT-2004-506078, and by the National Science Council of Taiwan under the grant numbers NSC96-2112-M002-020-MY3 and NSC96-2811-M002-026.

\section{References}

1. P. Hägler, PoS LAT2007, 013 (2007) [arXiv:hep-lat/0711.0819]

2. M. Göckeler, et al. [QCDSF Collaboration], Phys. Rev. Lett. 92, 042002 (2004) [arXiv:hep-ph/0304249]; P. Hägler, et al. [LHPC Collaboration], Phys. Rev. D 68, 034505 (2003) [arXiv:hep-lat/0304018]

3. M.A. Clark, PoS LAT2006, 004 (2006) [arXiv:hep-lat/0610048]

4. T. Bakeyev, et al. [QCDSF-UKQCD Collaboration], Phys. Lett. B 580, 197 (2004) [arXiv:hep-lat/0305014]

5. M. Göckeler, et al., PoS LAT2007, 129 (2007) [arXiv:hep-lat/0712.0010]

6. A. Ali Khan, et al. [QCDSF-UKQCD Collaboration], Nucl. Phys. B 689, 175 (2004) [arXiv:hep-lat/0312030]

7. C. Aubin, et al. Phys. Rev. D 70, 094505 (2004) [arXiv:hep-lat/0402030]

8. M. Procura, T.R. Hemmert, W. Weise, Phys. Rev. D 69, 034505 (2004) [arXiv:hep-lat/0309020]

9. V. Bernard, Prog. Part. Nucl. Phys. 60, 82 (2008) [arXiv: hep-ph/0706.0312]

10. A. Ali Khan, et al. [QCDSF Collaboration], Phys. Rev. D 74, 094508 (2006) [arXiv:hep-lat/0603028]

11. T.R. Hemmert, M. Procura, W. Weise, Phys. Rev. D 68, 075009 (2003) [arXiv:hep-lat/0303002]

12. M. Göckeler, et al. [QCDSF/UKQCD Collaboration], PoS LAT2007, 161 (2007) [arXiv: hep-lat/0710.2159]

13. R.G. Edwards, et al. [LHPC Collaboration], Phys. Rev. Lett. 96, 052001 (2006) [arXiv:hep-lat/0510062]

14. M.K. Jones, et al. [Jefferson Lab Hall A Collaboration], Phys. Rev. Lett. 84, 1398 (2000) [nucl-ex/9910005]; O. Gayou, et al. [Jefferson Lab Hall A Collaboration], Phys. Rev. Lett. 88, 092301 (2002) [arXiv:nucl-ex/0111010]

15. V. Punjabi, et al. [Jefferson Lab Hall A Collaboration], Phys. Rev. C 71, 055202 (2005); Erratum: Phys. Rev. C 71, 069902 (2005) [arXiv:nucl-ex/0501018]

16. M. Diehl, et al., Eur. Phys. J. C 39, 1 (2005) [arXiv:hep-ph/0408173]

17. T.R. Hemmert, W. Weise, Eur. Phys. J. A 15, 487 (2002) [arXiv:hep-lat/0204005]

18. M. Göckeler, et al. [QCDSF Collaboration], Phys. Rev. D 71, 034508 (2005) [arXiv: hep-lat/0303019]

19. G. Martinelli, C. Pittori, C.T. Sachrajda, M. Testa, A. Vladikas, Nucl. Phys. B 445, 81 (1995) [arXiv:hep-lat/9411010]; M. Göckeler, et al., Nucl. Phys. B 544, 699 (1999) [arXiv: hep-lat/9807044]

20. M. Göckeler, et al. [QCDSF Collaboration], Phys. Rev. D 73, 014513 (2006)

[arXiv : hep-ph/0502212] 
21. W. Detmold, et al., Phys. Rev. Lett. 87, 172001 (2001) [arXiv:hep-lat/0103006]

22. M. Dorati, T.A. Gail, T.R. Hemmert [arXiv:nucl-th/0703073]

23. D. Brömmel, et al. [QCDSF-UKQCD Collaboration], PoS LAT2007, 158 (2007) [arXiv:hep-lat/0710.1534]

24. M. Göckeler, et al. [QCDSF Collaboration], Phys. Rev. Lett. 98, 222001 (2007) [arXiv:hep-lat/0612032]

25. D. Brömmel, et al. [QCDSF/UKQCD Collaboration], Eur. Phys. J. C 51, 335 (2007) [arXiv:hep-lat/0608021]

26. D. Brömmel, et al. [QCDSF Collaboration] [arXiv:hep-lat/0708.2249] 